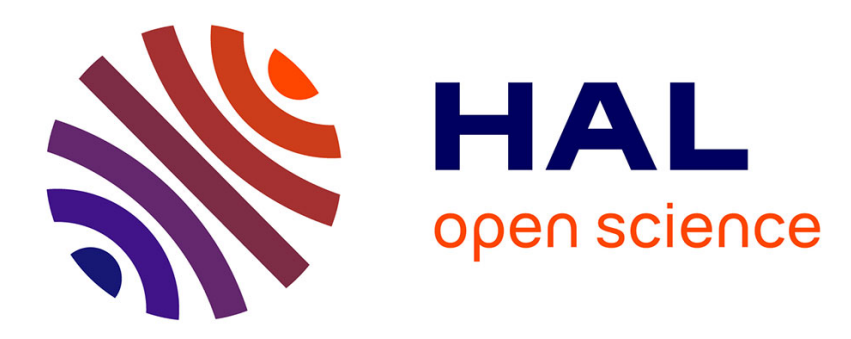

\title{
L'hélicoptère auxiliaire du reboiseur de montagne
}

\author{
A. Batias
}

\section{To cite this version:}

A. Batias. L'hélicoptère auxiliaire du reboiseur de montagne. Revue forestière française, 1961, 1, pp.25-30. 10.4267/2042/24313 . hal-03386228

\section{HAL Id: hal-03386228 \\ https://hal.science/hal-03386228}

Submitted on 19 Oct 2021

HAL is a multi-disciplinary open access archive for the deposit and dissemination of scientific research documents, whether they are published or not. The documents may come from teaching and research institutions in France or abroad, or from public or private research centers.
L'archive ouverte pluridisciplinaire HAL, est destinée au dépôt et à la diffusion de documents scientifiques de niveau recherche, publiés ou non, émanant des établissements d'enseignement et de recherche français ou étrangers, des laboratoires publics ou privés. 


\title{
L'HÉLICOPTËRE AUXILIAIRE DU REBOISEUR DE MONTAGNE
}

\author{
PAR \\ A. BATIAS \\ Ingénieur principal des Eaux et Forêts à Chambéry
}

Les exploits accomplis en montagne par les pilotes d'hélicoptères pour exécuter des missions de sauvetage, en particulier, en vue de porter secours aux alpinistes et aux skieurs en péril, notamment dans le Massif du Mont-Blanc, sont bien connus du grand public.

Cet appareil maniable et rapide, peut rendre de grands services pour la mise en valeur des montagnes d'accès difficile. Dans le numéro de mai 1959, F. Huin attirait l'attention des lecteurs de la Revue, sur les réalisations effectuées pour la construction et l'équipement des chalets d'alpages, de. halles à bétail et de baraquements (“ Transports en Montagne »-Revue forestière, n 2 de 1959). En Savoie, les Services Départementaux de la Reconstruction utilisèrent également l'hélicoptère pour reconstruire un chalet incendié pendant la guerre, sur la montagne de Margériaz, dans les Beauges.

Pour repeupler les lacs isolés de montagne, dans les Alpes et les Pyrénées, des hélicoptères furent également employés avec succès par les Fédérations de Pêche de l'Isère et de l'Ariège. Par la suite, P. Chimits perfectionna la technique en larguant directement les alevins en vol à faible hauteur, pour économiser les pertes de temps à l'atterrissage et à l'envol.

Ce mode de transport aérien permit également aux services forestiers de 1'Isère et de la Savoie, de régler un litige topographique entre deux commune limitrophes des deux départements. Les Maires, les Représentants des Services cadastraux et les Forestiers ont $\mathrm{pu}$, sur le terrain très accidenté du Mont Granier, vérifier une limite que des documents topographiques incertains ne permettaient pas d'établir.

$\mathrm{Pa}:$ suite de la dépopulation des campagnes, les alpages communaux, dans la zone subalpine de la Savoie (Beauges) sont abandonnés peu à peu. S’ils sont situés en dessous de la limite altitudinale 
des résineux, leur reboisement s'impose. Mais les difficultés de transport sont énormes, puisque ce sont précisément ces raisons qui les ont fait délaisser par les montagnards. Bien souvent ils ne sont accessibles que par d'étroits chemins muletiers

La solution qui consiste à construire un chemin ( jeepable ) à faible largeur et à forte déclivité sur plusieurs kilomètres, si séduisante soit-elle, de prime abord, est extrêmement coûteuse.

Non seulement le prix de revient de ces chemins est élevé $10000 \mathrm{NF}$ par kilomètre au minimum - mais leur entretien ultérieur est également fort onéreux. Construits sommairement sur de

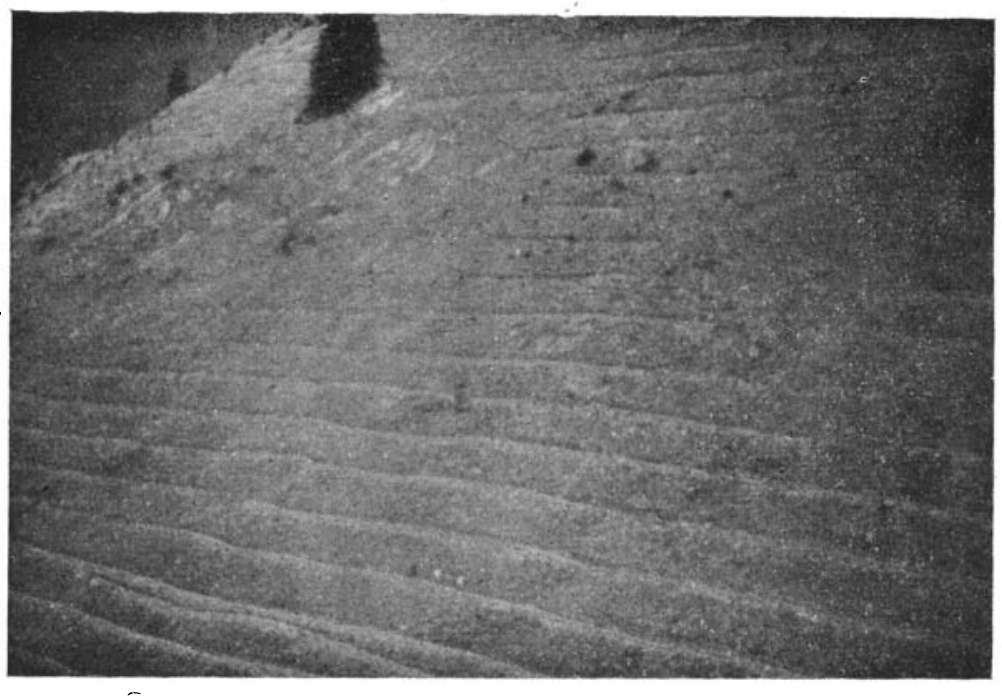

1 - Vue aérienne des banquettes établies pour les plantations des alpages de Courtace.

(Cliché Gallet.)

mauvais terrains ayant des pentes transversales élevées, sous un climat rigoureux, non seulement par la température mais aussi par. l'abondance des précipitations, il faut, chaque année, déblayer d'importants éboulements.

Pour quel usage: desservir un alpage pendant les trois années nécessaires à son boisement et attendre 50 ans que les premiers produits d'éclaircie puissent être mis en vente.

Les normes de tels chemins ne correspondant pas aux exigences des routes forestières modernes, ne permettront probablement pas de les utiliser pour la vidange des forêts créées.

C'est pourquoi, l'expérience réalisée cette année par « Les Pépinières et Reboisement Louis GALlét à La Chaise-Dieu (HauteLoire) ) nous paraît susceptible d'intéresser les lecteurs de la Revue: 
Il s'agissait de boiser d'anciens pâturages achetés par l'Etat et englobés dans la série de reboisement du Haut-Chéran, périmètre du Fier. Ils sont situés dans les hautes Beauges, sur le territoire de la commune de Jarsy, entre 1380 et $1680 \mathrm{~m}$ d'altitude, sur sol argilo-calcaire, valanginien et hauterivien d'excellente qualité pour un boisement mais très défavorable à l'établissement d'une chaussée solide.

L'enrésinement effectué grâce à un contrat du F.F.N., prévoit la mise en terre, en trois ans $(1960,1961,1962)$ sur une superficie de 31,26 ha de 73164 épicéas $2+2$ et 3120 sapins $2+2$. Les plantations sont faites suivant des lignes parallèles aux courbes de niveau, espacées de 2 mètres. Dans chaque ligne, les tiges sont placées en quinconce à $1,90 \mathrm{~m}$ d'intervalle. Quand la pente est supérieure à $70 \%$ (12 ha environ), les plantations ne sont exécutées qu'une ligne sur trois, sur banquettes de $0,40 \mathrm{~m}$ de largeur. Les lignes vides s'opposent ainsi à la reptation de la neige. Quelques potets sont prévus dans les parties déjà boisées en feuillus pour l'introduction du sapin pectiné. Le devis annexé au contrat envisage en outre, une fumure par apport d'engrais à raison de $225 \mathrm{gr}$ de scories de déphosphoration par" plant.

Tous les travaux de préparation du sol sont exécutés facilement au cours de l'été, nais la plantation elle-même doit être réalisée à l'automne dans un temps extrêmement bref. Pour mettre en place les plants, il est nécessaire d'atteindre la fin de la sève (fin septembre) et les travaux doivent être terminés avant la neige qui peut recouvrir ces terrains d'altitude élevée de bonne heure, quelquefois avant la fin octobre.

Les équipes de planteurs disposent d'un mois environ pour exécuter le travail, ce qui implique un transport de plants très rapide.

La maison GALLET, adjudicataire du travail, produit les épicéas et sapins dans sa pépinière de La Chaise-Dieu (Haute-Loire) à $220 \mathrm{~km}$ de l'extrémité de la route forestière accessible aux camions desservant la série du Haut Chéran à $950 \mathrm{~m}$ d'altitude.

Cette route est terminée par une plateforme circulaire en bordure d 11 torrent du Chéran et à $700 \mathrm{~m}$ en dessous d'un chalet forestier de Coutarce qui permet de loger le personnel et d'entreposer matériel, plants et engrais.

La distance à vol d'oiseau de la plateforme au chalet est de $3 \mathrm{~km}$ Un chemin étroit, long de $7 \mathrm{~km}$ et de mauvaise qualité permet à une jeep de relier la route forestière au chantier. Constitué tantôt par de petits rochers et 'des pierres roulantes, tantôt par un sol argileux très gras, il n'est circulable que par temps sec. Son étroitesse et le faible rayon de courbure des virages ne permettent pas de mettre une remorque à la jeep. Le transport automobile ne peut être réalisé qu'à une cadence de $300 \mathrm{~kg}$ par voyage, ce qui n'est possible qu'une fois par jour. 
Le tonnage total à transporter s'élevait à:
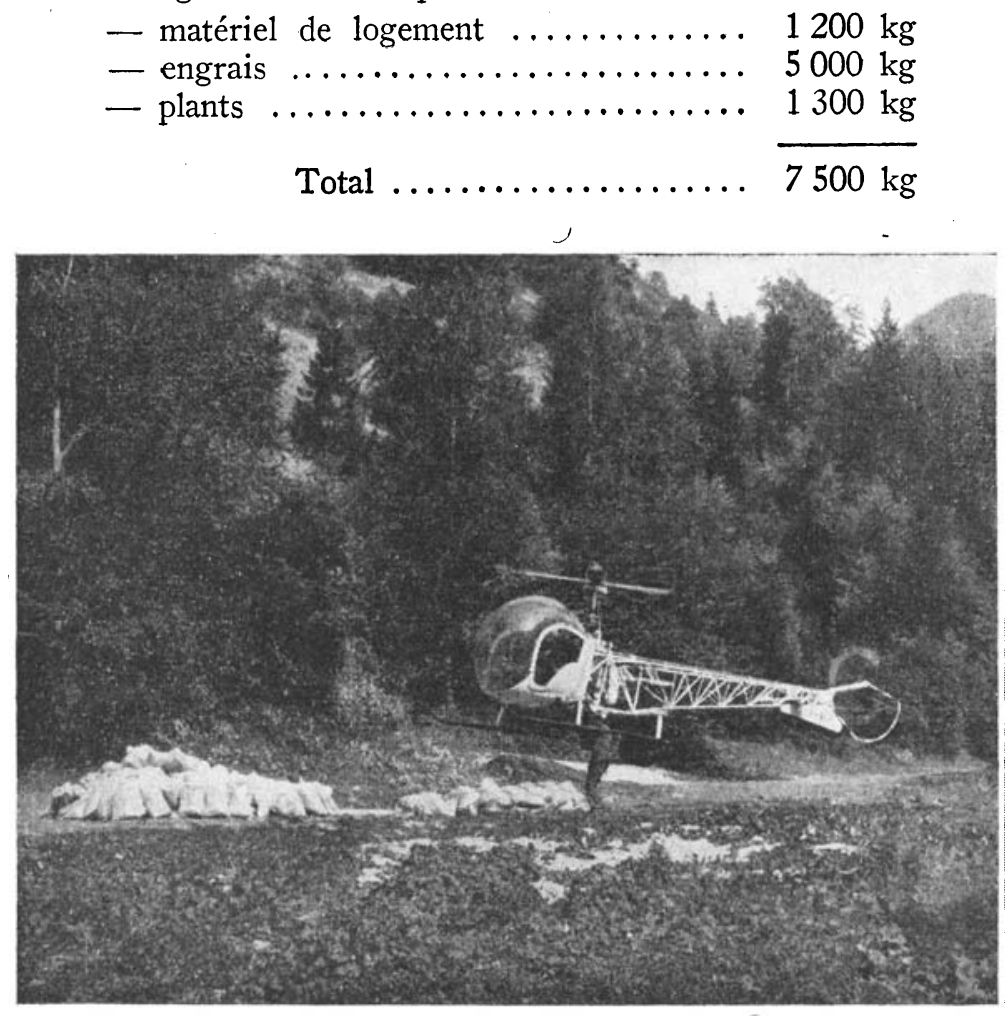

2 - Atterrissage de l'hélicoptère à la base de départ. Sur la gauche, sacs en polyéthylène contenant les plants. Au centre, sous l'appareil sacs de scories. (Cliché Gyrafrique.)

représentant 25 voyages en jeep sans remorque et un coût de $1000 \mathrm{NF}$ pour une durée de transport de deux mois, compte tenu du mauvais temps.

Le programme prévu était irréalisable.

M. Gallet demanda les services de la Société Gyrafrique qui possède une base d'hélicoptères type Bell 47 G.2 à Albertville, $\mathrm{La}$ plateforme terminale de la route forestière constituait une aire de départ de $50 \mathrm{~m}^{2}$ où tout le matériel était approvisionné. Une aire d'atterrissage de $20 \mathrm{~m}^{2}$ fut très facilement aménagée dans le pâturage devant le chalet de Coutarce.

GYRAFRIQUe mit à la disposition de l'entrepreneur de boisement le 29 septembre à 7 heures du matin, un hélicoptère et un équipage comprenant un pilote et un mécanicien. L'entreprise fournissait deux équipes de deux hommes, pour procéder à la confection des 
charges dans la zone de départ et à leur réception à la zone d'arrivée. L'emport de charges était fixé entre 200 et $250 \mathrm{~kg}$ le matin du travail en fonction de l'aérologie locale.

Le prix était facturé à l'heure de vol, sur la base de $650 \mathrm{NF}$ l'heure de vol effective comprenant le temps s'écoulant entre le moment où le rotor de l'appareil tourne et le moment où il s'arrête. Ce prix comprenait toutes taxes et prestations de service, notamment la fourniture des filets et élingues nécessaires au transport.

Le devis prévoyait une durée de transport de 5 heures.

.L'entrepreneur approvisionna les engrais la veille du transport, mais il n'amena le matériel et les plants que le matin même.

Le programme prévu fut réalisé parfaitement entre 7 heures et 12 heures. Les charges furent transportées dans des filets fixés par des élingues à la partie inférieure de l'hélicoptère. Un jeu de six filets permit aux équipes de départ et d’arrivée de préparer les charges et de les enlever pendant les voyages de l'appareil.

Celui-ci se posait toutes les cinq rotations pour faire le plein l'essence et pour décharger les filets redescendus de la montagne.

Le matériel de logement fut transporté en 24 minutes, par 6 voyages. Pour 5 tonnes de scories, le pilote dut effectuer 25 rotations de 5 minutes, soit 2 heures 5 minutes.

Quant aux plants, ils étaient emballés dans des sacs en polyéthylène. Ne disposant pas de filets spéciaux pour le transport de charges volumineuses, le poids par voyage dut être réduit à $130 \mathrm{~kg}$. Pour compenser cette perte, le trajet put être raccourci en effectuant des montées verticales, ce qui ramena la durée de la rotation à 4 minutes, soit 40 minutes au total.

I a clurée de transport du matériel, des engrais et des plants correspondit à 3,09 heures de vol effectif.

Le prix de revient s'éleva à $2057 \mathrm{NF}$ pour effectuer une plantation de 24400 épicéas et 1200 sapins, soit 0,08 NF par plant.

Si l'on compare ce coût avec le transport en jeep, l'opération ne semble pas rentable (2057 NF contre $1000 \mathrm{NF}$ ). En réalité, le transport automobile à une cadence de 25 voyages en deux mois aurait nécessité sur un parcours de $7 \mathrm{~km}$ des travaux d'entretien d'au moins $1000 \mathrm{NF}$ et probablement $1500 \mathrm{NF}$.

Nous pensons donc qu'au point de vue strictement financier, l'emploi de l'hélicoptère ne fut pas plus coûteux que celui d'une jeep. Il se solda même par un bénéfice, puisqu'il ne fut pas utile de mettre en jauge les plants à l'extrémité de la route d'accès.

En ce qui concerne les conditions techniques du boisement, l'emploi de l'hélicoptère fut particulièrement bénéfique en raison:

- de la réduction des manutentions,

- de la douceur de ce moyen de transport,

- de la diminution du temps écoulé entre l'arrachage en pépinière et la mise en place sur le chantier. 
Les plants arrachés le 28 septembre à la Chaise-Dieu étaient en totalité sur les lieux de boisement le 29 septembre à midi.

- ¿de la sécurité de l'approvisionnement.

C'est en définitive cette sécurité qui justifie le mieux l'emploi de l'hélicoptère pour transporter les plants pour les boisements d'alpages.

Les intempéries de l'automne devaient rapidement apporter une justification spectaculaire à l'expérience tentée par M. Gallet.

Dès le 30 septembre, une tornade d'une grande violence s'abattit sur les hautes Beauges, le Chéran démesurément enflé sortit de son lit et coupa la route forestière qui fut en outre obstruée en plusieurs endroits par d'importants éboulements. Les résineux n'auraient donc pas pu être transportés par jeep sur le chantier.

Les planteurs installés au chalet de Coutarce n'auraient pu travailler et les plants restés en bas en bordure du Chéran auraient vraisemblablement été perdus.

$\mathrm{Au}$ contraire, grâce à l'hélicoptère, malgré une saison particulièrement défavorable, les plantations prévues au contrat furent réalisées dans d'excellentes conditions.

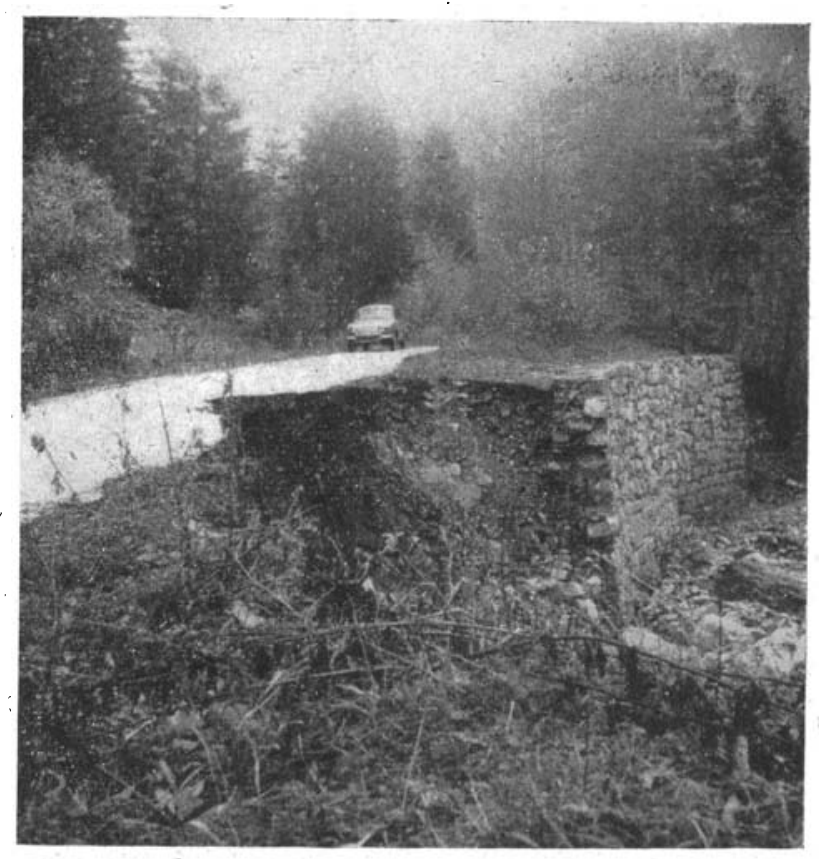

3 - Coupure de la route forestière d'accès par la crue du Chéran, le 30 septembre 1960, au lendemain de l'opération « Hélicoptère 》.

(Cliché Roux.) 